RAD Conference Proceedings, vol. 4, pp. 72-75, 2020

ISSN 2466-4626 (online) | DOI: 10.21175/RadProc.2020.15

www.rad-proceedings.org

\title{
ASSESSING THE ROLE OF VMAT RELATIVE TO IMRT FOR PATIENTS AFTER PROSTATECTOMY IN TERMS OF SPARING PELVIC BONES
}

\author{
Maria Poncyljusz ${ }^{*}$, Wojciech Bulski² \\ ${ }^{1}$ The Central Clinical Hospital of the Ministry of the Interior and Administration in Warsaw, Poland \\ ${ }^{2}$ The Maria Sklodowska-Curie National Research Institute of Oncology, Warsaw, Poland
}

\begin{abstract}
For postprostatectomy patients at higher risk of nodal involvement the irradiation of pelvic lymph nodes may improve the therapeutic ratio. However, whole pelvic radiotherapy results in increased doses delivered to the pelvic bones' marrow and other OARs. The aim of this study was to compare IMRT and VMAT techniques in terms of sparing of pelvic bones. The VMAT and IMRT plans were created for ten patients. Treatment plans were generated for prostate bed (PTV1) and pelvic lymph nodes (PTV2). The delivered mean dose to the sum of PTV1 and PTV2 was $46 \mathrm{~Gy}$ in 23 fractions and additionally a mean dose of $18 \mathrm{~Gy}$ in 9 fractions was prescribed to PTV1. The target coverage and the OARs sparing were compared across techniques. The following OARs were delineated: pelvic bones, bowel bag, rectum and bladder. The following dose volume parameters were compared using the Wilcoxon test: pelvic bones V3oGy[\%], V4OGy[\%], the bowel bag V3oGy[cc], V45Gy[cc], rectum V4oGy[\%], V5oGy[\%], V6oGy[\%] and bladder V4OGy[\%], V5oGy[\%], V6oGy[\%]. The target coverage in VMAT and IMRT plans was comparable. The value of PTV1 V95\% and PTV2 V95\% were both >99\%. The VMAT plans result in decrease of pelvic bones V3oGy[\%] and significant increase of the following parameters: rectum V6oGy[\%], bladder V6oGy[\%] and bowel bag V3oGy[cc]. Differences between values of V4OGy[\%] and V5OGy[\%] for bladder and rectum across mentioned techniques were statistically not significant. A comparison between VMAT and IMRT techniques showed, that the VMAT technique reduces the delivered dose to pelvic bones and thus also to red marrow. However, IMRT provided better sparing at higher doses for rectum, bladder and bowel bag. These results should be taken into consideration when VMAT and IMRT techniques being used in the whole pelvic radiotherapy of patients after radical prostatectomy.
\end{abstract}

Key words: Bones marrow, haematological toxicity, prostate cancer, radiotherapy, whole pelvic radiotherapy

\section{INTRODUCTION}

The potentially eradicating nodal micrometastases after irradiation of the pelvic lymph nodes may improve the treatment outcome for post-prostatectomy patients with a higher risk of nodal involvement [1]. The target including prostate bed and pelvic lymph nodes is irregularly shaped and surrounded by a significant extent of normal tissues when compared to the irradiation of prostate bed only. The irradiation of this target may lead to an increase of doses delivered to surrounding organ at risk (OARs) including the pelvic bones. The dose to the pelvic bones is a surrogate of the dose delivered to the bone marrow, which is an important factor in the treatment process. Sini et al. reported that increased pelvic bones $\mathrm{V} 40 \mathrm{~Gy}[\mathrm{cc}]$ is associated with an increased risk of acute G3 and late G2 lymphopenia [2].

The use of advanced radiotherapy techniques like volumetric arc therapy (VMAT) and intensity modulated radiotherapy (IMRT) provide improved OARs sparing, while maintaining a similar level of the target coverage compared to conformal radiotherapy (3D-CRT) [3-9]. The dose distributions are different for plan generated by VMAT and IMRT techniques due to the use of various geometrical parameters in the plan optimisation (gantry angle, MLC shape etc.). Some authors reported the differences between dosimetric parameters of dose distribution for VMAT and IMRT plans. Davidson et al. compared the IMRT, VMAT and HT (Helical Tomotherapy) plans created for prostate cancer patients [10]. However, the dose delivered to the pelvic bones was not taken into account by these authors.

It is anticipated that the use of VMAT technique results in different DVH parameters for pelvic bones and the other OARs than IMRT.

In this study the role of VMAT was assessed relative to IMRT. The doses delivered to the pelvic bones and the other OARs were considered. The differences in the dose distributions for these techniques were analysed. The aim of this study was to assess feasibility of dynamic techniques to spare the pelvic bones and other OARs.

\section{MATERIALS AND METHODS}

The data set for this study consists of treatment plans for 10 patients treated between 2012 and 2014 . The VMAT and IMRT plans were created for each patient. Only IMRT plans were used in clinical treatment.

The clinical indications for radiotherapy are presented in Table 1.

*mjaponc@gmail.com 
Table 1. Clinical indications for radiotherapy

\begin{tabular}{|l|l|}
\hline tumour stage & $\begin{array}{l}\mathrm{pT} \text { 3a/b - exceeding the } \\
\text { prostate and/or } \\
\text { infiltration of the } \\
\text { seminal vesicle }\end{array}$ \\
\hline \multicolumn{2}{|l|}{ positive surgical margin (R1) } \\
\hline \multicolumn{2}{|l|}{ lymph node involvement $\mathrm{N}(+)$} \\
\hline PSA & $\begin{array}{l}>0.2 \mathrm{ng} / \mathrm{ml} \text { at least } 5 \\
\text { weeks after surgery }\end{array}$ \\
\hline
\end{tabular}

The patients underwent a planning CT scans in the supine position with a knee support and with their hands resting on their chest. Planning CT scans were obtained from the mid-level L4 up to $5 \mathrm{~cm}$ below the ischial tuberosity. The scans were taken using computed tomography with $3 \mathrm{~mm}$ slice thickness. The patients were asked to drink $500 \mathrm{ml}$ of water 30 minutes prior to the CT scan and then before each irradiation session.

The prostate bed was contoured as CTV1 depending on the diagnosis, with or without the seminal vesicles (classification T3a - covered base of seminal vesicle, classification $T_{3} b$ - covered $2 / 3$ of seminal vesicle). PTV1 was grown by giving isotropic $1 \mathrm{~cm}$ margin to CTV1.

The pelvic lymph nodes were contoured starting from the $\mathrm{L} 5 / \mathrm{S} 1$ level up to the upper edge of the symphysis pubis to represent CTV2. The external iliac nodes were drawn up to the top of the femoral heads. CTV2 was then grown isotropically by $7 \mathrm{~mm}$ to create PTV2.

The pelvic bones were delineated from the level of the ischial tuberosities up to the tops of upper iliac, including the femurs. The rectum, the bladder, the bowel bag and the left and right femurs (femur L/R) were drawn according to the recommendations of the RTOG pelvis atlas [11]. The bowel bag was cropped from PTV1 and PTV2 with no margin.

The treatment was delivered in two phases. Initially, the prescribed mean dose of $46 \mathrm{~Gy}$ with a fractionation regime of $2 \mathrm{~Gy}$ per fraction was delivered to PTV1 and PTV2. During the second part PTV1 was irradiated to $18 \mathrm{~Gy}$ in 9 fractions.

All treatment plans were designed using a commercial treatment planning system (Eclipse ver. 6.5, Varian Medical Systems, Inc.) and AAA algorithm (Anisotropic Analytical Algorithm, Ver. 10.0.28).

VMAT plans were optimised for two arcs (rotation of $356^{\circ}$, 1st arc: from $178^{\circ}$ to $182^{\circ} \mathrm{CCW}$, collimator $30^{\circ}$ and 2nd arc : from $182^{\circ}$ to $178^{\circ} \mathrm{CW}$, collimator $330^{\circ}$ ) for a Clinac_2300 equipped with Millennium120 MLC (120 leaves with a resolution at isocentre of $5 \mathrm{~mm}$ for the inner $20 \mathrm{~cm}$ and $10 \mathrm{~mm}$ for the outer $2 \times 10 \mathrm{~cm}$ ) and a photon beam energy of 6MV.

Sliding window IMRT plans were created using 5 photon beams with an energy of $6 \mathrm{MV}$ (fixed gantry angles of $\left.\mathrm{O}^{0}, 72^{\circ}, 144^{\circ}, 216^{\circ}, 288^{\circ}\right)$. Small modification to the gantry angle was allowed in order to spare the femoral heads. Collimator angle of $3^{0}$ was chosen to minimize the overlap of interleaf leakage.

For consistency, VMAT plans were designed using the same normal tissue constraints as the IMRT plans. The PTV planning objective was to deliver more than $95 \%$ of the dose to a minimum of $98 \%$ of the target volume. The dose volume histograms were evaluated for both techniques for PTV and organs at risk alternatively. According to a "Quantitative Analysis of Normal Tissue Effects in the Clinic" study [12] the values listed in Table 2 were compared between VMAT and IMRT. Wilcoxon signed-rank test was run for all critical organs and p-values were calculated.

Table 2. The dose distribution indices used for comparison

\begin{tabular}{|l|l|}
\hline OAR & Analysed value \\
\hline pelvic bones & $\mathrm{V}_{30 \mathrm{OGy}}[\%], \mathrm{V}_{4 \mathrm{OGy}}[\%]$ \\
bladder & $\mathrm{V}_{40 \mathrm{OGy}}[\%], \mathrm{V}_{50 \mathrm{OG}}[\%], \mathrm{V}_{60 \mathrm{~Gy}}[\%]$ \\
rectum & $\mathrm{V}_{40 \mathrm{~Gy}}[\%], \mathrm{V}_{50 \mathrm{OG}}[\%], \mathrm{V}_{60 \mathrm{~Gy}}[\%]$ \\
bowel bag & $\mathrm{V}_{30 \mathrm{OGy}}[\mathrm{cc}], \mathrm{V}_{45 \mathrm{~Gy}}[\mathrm{cc}]$ \\
\hline
\end{tabular}

\section{RESULTS}

The dosimetric quantities of dose distribution in the PTV for VMAT and IMRT techniques were comparable. For both techniques for all patients the value of V95\% was found to be more than $99 \%$ in PTV1 and PTV2.
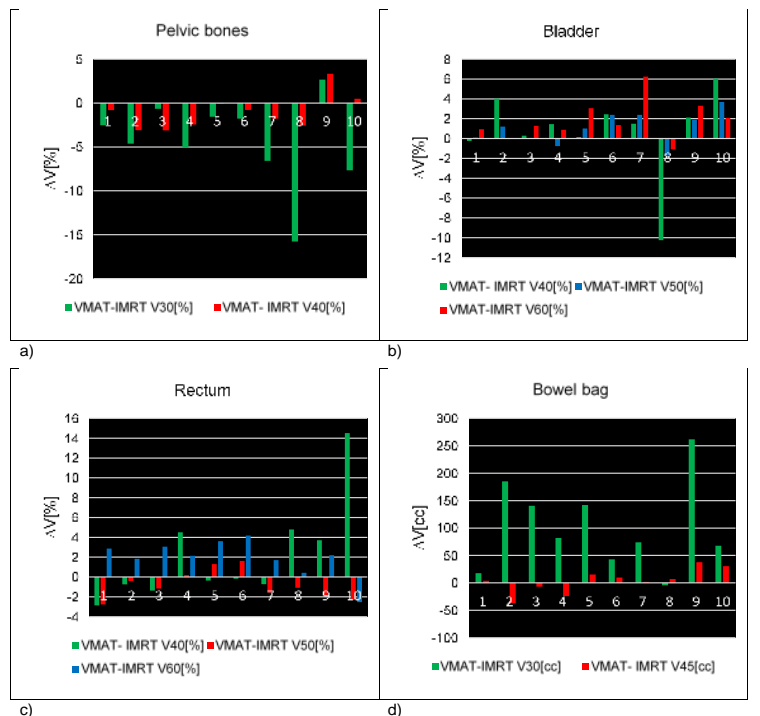

Figure 1. The differences in analysed values for pelvic bones a), bladder b), rectum c), bowel bag d) between techniques for all ten patients

Figure 1 demonstrates the differences in sparing of OARs for both techniques in terms of the dose distribution indices described in Table 2. 
VMAT shows better sparing of the bone marrow as a result of significant reduction of pelvic bones V3oGy[\%]. This technique lead to considerable increase of the following dosimetric factors: bladder V6oGy[\%], rectum V6oGy[\%] and bowel bag V3oGy[cc]. The differences between values of V40Gy[\%] and V5OGy[\%] for the rectum, the bladder and the bowel bag $\mathrm{V} 45 \mathrm{~Gy}[\mathrm{cc}]$ across mentioned techniques were not statistically significant (Table 3).

Table 3. The p-values obtained using the Wilcoxon signedrank test to compare VMAT and IMRT; (NS - not statistically significant)

\begin{tabular}{|c|c|c|}
\hline OAR & $\begin{array}{l}\text { Dose volume } \\
\text { parameter }\end{array}$ & $\begin{array}{l}\text { p-value } \\
\text { VMAT vs IMRT }\end{array}$ \\
\hline \multirow{2}{*}{ pelvic bones } & $\mathrm{V}_{3 \mathrm{OGy}}[\%]$ & 0.022 \\
\hline & $\mathrm{V}_{4 \mathrm{OGy}}[\%]$ & NS \\
\hline \multirow{3}{*}{ bladder } & $\mathrm{V}_{4 \mathrm{OGy}}[\%]$ & NS \\
\hline & $\mathrm{V}_{5 \mathrm{OGy}}[\%]$ & NS \\
\hline & $\mathrm{V}_{60 G y}[\%]$ & 0.017 \\
\hline \multirow{3}{*}{ rectum } & $\mathrm{V}_{4 \mathrm{OGy}}[\%]$ & NS \\
\hline & $\mathrm{V}_{5 \mathrm{OGy}}[\%]$ & NS \\
\hline & $\mathrm{V}_{60 G y}[\%]$ & 0.028 \\
\hline \multirow[t]{2}{*}{ bowel bag } & $\mathrm{V}_{3 \mathrm{OGy}}[\mathrm{cc}]$ & 0.007 \\
\hline & $\mathrm{V}_{45 \mathrm{~Gy}}[\mathrm{cc}]$ & NS \\
\hline
\end{tabular}

\section{DISCUSSION}

Pelvic flat bones with active bone marrow are regarded as an important critical organ during whole pelvic radiotherapy. The irradiation of pelvic bones can influence the hemopoetic function of red bone marrow. Mell et al. [13] reported a decrease of haematological toxicity after reducing the dose delivered to the bone marrow for patient with rectal cancer. Scini et al. [2] recommended the pelvic bones $\mathrm{V} 4 \mathrm{OGy}[\mathrm{cc}]$ as predictor in red bone marrow sparing. Therefore, the main goal of this study was to compare VMAT and IMRT in terms of pelvic bones sparing.

In this study the difference between the values of the pelvic bones V40Gy[\%] for VMAT and IMRT plans was not statistically significant. Hence, it could be anticipated that VMAT technique is comparable to IMRT in terms of the red bone marrow. However, the value of the pelvic bones V30Gy[\%] in IMRT plans was higher than in VMAT plans. This result is the major advantage of VMAT technique. It should be noted that 5 photon beams plans were used in IMRT technique. A larger or lower number of fields may, of course, yield different results. The 5 -field IMRT technique was used in the clinic to avoid prolonged irradiation time. It is known that the long time of irradiation increases the risk of motion during the fraction and causes a change in bladder filling, which adversely affects the accuracy of treatment. A greater number of beams also results in the deposition of lower doses in a larger area of normal tissues.

When it comes to the bladder and rectum QANTEC [12] recommends the following constraints: V65Gy $<50 \%$ and V50Gy $<50 \%$, respectively. Dynamic techniques allow to reduce the volumes receiving higher doses (more than 50Gy) for postprostatectomy patient at higher risk of nodal involvement [14]. Therefore, in this investigation the value of $\mathrm{V} 4 \mathrm{OGy}[\%]$, V5oGy[\%] and V6oGy[\%] for bladder and rectum were analysed.

Statistically significant differences were obtained only for value V6oGy[\%]. This result is in line with obtained by Davidson et al. [10] which shows the values of $\mathrm{V} 60 \mathrm{~Gy}[\%]$ for bladder and rectum were higher in VMAT plans.

Analysis of the bowel bag $\mathrm{V} 45[\mathrm{cc}]$ (constraint recommended by QUANTEC [12]) shows that the differences between techniques were not statistically significant. In contrast to the results obtained for the bowel bag V30Gy[cc] the volumes of the bowel bag receiving 30Gy and more were lower in IMRT plans.

The above results should be taken into account when selecting the appropriate technique for patients whose clinical indications require additional dose limitation in mentioned OARs.

\section{CONCLUSION}

The two-arc VMAT and 5-field IMRT techniques were compared in the study. VMAT technique offers delivering of lower doses to the pelvic bones what results better sparing of bone marrow. On the other hand, for complicated and large PTV that included prostate bed and lymph nodes IMRT proved better sparing of bladder, rectum and bowel bag.

\section{REFERENCES}

1. M.T. Spiotto, S.L. Hancock, C.R. King, "Radiotherapy after prostatectomy: improved biochemical relapse-free survival with whole pelvic compared with prostate bed only for high-risk patients," Int. J. Radiat. Oncol. Biol. Phys., vol. 69, no.1, pp. 54-61, Sep. 2007. http://doi.org/10.1016/j.ijrobp.2007.02.035 PMid: 17459606

2. C. Sini, C. Fiorino, L. Perna, et al. "Dose-volume effects for pelvic bone marrow in predicting haematological toxicity in prostate cancer radiotherapy with pelvic node irradiation," Radiother. Oncol., vol. 118, no.1, pp. 79-84, Jan. 2016.

http://doi.org/10.1016/j.radonc.2015.11.020 PMid: 26702990

3. Ch. Kusumoto, S. Ohira, M. Miyazaki, et al. "Effect of various methods for rectum delineation on relative and absolute dose-volume histograms for prostate IMRT treatment planning," Medical Dosimetry, vol. 41, no. 2, pp. 136-141, Jun. 2016. http://doi.org/10.1016/j.meddos.2015.11.001

4. S. Sutani, T. Ohashi, M. Sakayori, et al. "Comparison of genitourinary and gastrointestinal toxicity among four radiotherapy modalities for prostate cancer: Conventional radiotherapy, intensity-modulated radiotherapy, and permanent iodine-125 implantation with or without external beam radiotherapy," 
Radiother. Oncol., vol. 117, no.2, pp. 270-276, Nov. 2015.

http://doi.org/10.1016/j.radonc.2015.08.019 PMid: 26318662

5. C. J. Neill, "Dosimetric comparison of intensitymodulated solutions for intact prostate cancer," Medical Dosimetry, vol. 39, no. 4, pp. 366-373, 2014. http://doi.org/10.1016/j.meddos.2014.06.006

6. E. A. Mellon, K. Javedan, T. J. Strom, et al "A dosimetric comparison of volumetric modulated arc therapy with step-and-shoot intensity modulated radiation therapy for prostate cancer," Practical Radiation Oncology, vol. 5, no. 1, pp. 11-15, Jan. 2015 http://doi.org/10.1016/j.prro.2014.03.003 PMid: 25413432

7. R. Wortel, L. Incrocci, F. Pos, et al. "Image-guided IMRT reduces late toxicity compared to 3D-CRT for prostate cancer," Radiother. Oncol., vol. 119, pp. S346-S347, Apr. 2016. http://doi.org/10.1016/So167-8140(16)31992-2

8. H. E. Carter, A. Martin, D. Schofield, et al. "A decision model to estimate the cost-effectiveness of intensity modulated radiation therapy (IMRT) compared to three dimensional conformal radiation therapy (3DCRT) in patients receiving radiotherapy to the prostate bed," Radiother. Oncol., vol. 112, no. 2, pp. 187-193, Aug. 2014. http://doi.org/10.1016/j.radonc.2014.03.020 PMid: 24929702

9. S. Katayama, T. Striecker, K. Kessel, et al. "Hypofractionated IMRT of the prostate bed after radical prostatectomy: acute toxicity in the PRIAMOS-1 Trial," Radiother. Oncol., vol. 90, no.4, pp. 926-933, Nov. 2014. http://doi.org/10.1016/j.ijrobp.2014.07.015 PMid: 25216858

10. M. Davidson, S. Blake, D. Batchelar, et al. "Assessing the role of volumetric modulated arc therapy (VMAT) relative to IMRT and helical tomotherapy in management of localized, locally advanced, and postoperative prostate cancer," Int. J. Radiat. Oncol. Biol. Phys., vol. 8o, no. 5, pp. 1550-1558, Aug. 2011. http://doi.org/10.1016/j.ijrobp.2010.10.024 PMid: 21543164

11. H. A. Gay, H. J. Barthold, E. O’Meara et al. "Pelvic Normal Tissue Contouring Guidelines for Radiation Therapy: A Radiation Therapy Oncology Group Consensus Panel Atlas," J. Radiat. Oncol. Biol. Phys., vol. 83, no. 3, pp. E353-E362, Jul. 2012. http://doi.org/10.1016/j.ijrobp.2012.01.023

12. L. B. Marks, E. D. Yorke, A. Jackson, et al. "Use of normal tissue complication probability models in the clinic,” Int. J. Radiat. Oncol. Biol. Phys., vol. 76, no. 3, pp. S10-S19, Mar. 2010.

http://doi.org/10.1016/j.ijrobp.2009.07.1754 PMid: 20171502 PMCid: PMC4041542

13. L. K. Mell, D. A. Schomas, J. K. Salama, et al. "Association between bone marrow dosimetric parameters and acute hematologic toxicity in anal cancer patient treated with concurrent chemotherapy and intensity-modulated radiotherapy," Int. J. Radiat. Oncol. Biol. Phys., vol. 70, no. 5, pp. 1431-1437, Apr. 2008.

http://doi.org/10.1016/j.ijrobp.2007.08.074 PMid: 17996390

14. F. Alongi, C. Fiorino, C. Cozzarini, et al. "IMRT significantly reduces acute toxicity of whole-pelvis irradiation in patients treated with post-operative adjuvant or salvage radiotherapy after radical prostatectomy," Radiother. Oncol., vol. 93, no. 2, pp. 207-212, Nov. 2009. http://doi.org/10.1016/j.radonc.2009.08.042 